\title{
Production and Diagnosis of Energetic Particles in FAST
}

M. Tardocchi 1), M. Nocente 1), A. Bruschi 1), D. Marocco 2), G. Calabrò 2), A. Cardinali 2), F. Crisanti 2), B. Esposito 2), L. Figini 1), G. Gorini 1), G. Grossetti 1), G. Grosso 1), M. Lontano 1), S. Nowak 1), F. Orsitto 2), U. Tartari 1) and O. Tudisco 2)

1) Associazione EURATOM-ENEA, IFP-CNR, Via R. Cozzi 53, 20125 Milano, Italy

2) Associazione EURATOM-ENEA, CR ENEA-Frascati, Via E.Fermi 45, Frascati (Roma), Italy

E-mail contact of main author: tardocchi@ifp.cnr.it

\begin{abstract}
The Fusion Advanced Study Torus (FAST) has been proposed as a possible European satellite facility to study in deuterium plasmas fast ion physics in conditions relevant to a burning plasma. Energetic ${ }^{3} \mathrm{He}$ particles, with dimensionless parameters close to those of the fusion-born alphas in ITER, can be produced in FAST via 30 MW power ICRH minority heating. This work provides a first assessment of the extent to which the ${ }^{3} \mathrm{He}$ fast ion population can be diagnosed in FAST with a set of dedicated confined fast particle diagnostics. Neutron Emission Spectroscopy (NES), Gamma-Ray Spectroscopy (GRS) and Collective Thomson Scattering (CTS) diagnostics have been reviewed with a description of the state-of-the-art hardware and a preliminary analysis of the required lines of sight. The results of the analysis, based on numerical simulations of the spatial and energetic particle distribution function of the ICRH-accelerated ions for the standard FAST H-mode scenario, suggest that NES and GRS measurements can provide an information on the anisotropy of the fast ${ }^{3} \mathrm{He}$ population and a 1 measurement of its effective tail temperature, with time resolutions in the range 20-100 ms. The proposed CTS diagnostic can measure the fast ion parallel and perpendicular temperature with a spatial resolution of $5-10 \mathrm{~cm}$ and a time resolution of $10 \mathrm{~ms}$. The paper provide a scientific basis for the predictions of FAST capability in the production and diagnosis of energetic ions.
\end{abstract}

\section{Introduction}

The Fusion Advanced Studies Torus (FAST) has been proposed as a possible European satellite facility with the aim of supporting the ITER program and investigating physical and technological issues relevant to DEMO [1]. FAST is a compact Tokamak $(\mathrm{R}=1.82 \mathrm{~m}, \mathrm{a}=0.64 \mathrm{~m})$ with high toroidal magnetic field (up to $8.5 \mathrm{~T}$ ) and plasma current (up to $8 \mathrm{MA}$ ), equipped with $30 \mathrm{MW}$ of additional heating in the form of Ion Cyclotron Resonance Heating (ICRH) [2]. Plasma edge/ELMs characteristics similar to those of ITER and a fusion gain of up to 1.5 are foreseen in the FAST Hmode scenario [1]. One of the main objective of FAST is the study, in deuterium plasmas, of fast ion physics in conditions relevant to a burning plasma. Fast ${ }^{3} \mathrm{He}$ particles with dimensionless parameters (normalized Larmor radius, $\rho_{\mathrm{H}}$, and plasma pressure, $\beta_{\mathrm{H}}$ ) close to those of the fusionborn alphas in ITER can be produced in FAST via ICRH minority heating [1,2]. This paper investigates the possibility of diagnosing ${ }^{3} \mathrm{He}$ fast ion populations in FAST with a set of dedicated diagnostics of confined fast particles: Neutron Emission Spectroscopy (NES), Gamma-Ray Spectroscopy (GRS) and Collective Thomson Scattering (CTS). Other diagnostics techniques of the fast ion population, like charge exchange recombination spectroscopy and lost fast ions probes, are also envisaged in FAST but they are not discussed in the present work. Taking as input numerical simulations of the spatial and energetic probability distribution function of the ICRHaccelerated particles [2], the corresponding spectra observed by the set of diagnostics have been simulated and the observables related to the fast ion tails have been analyzed, with the achievable spatial and temporal resolution.

\section{Energetic ion population produced by ICRH}

The FAST operating scenario considered for this study is the H-mode reference (Table 1, [1]). This scenario reaches the $\mathrm{L}$ to $\mathrm{H}$ mode power thresholds thanks to a large ICRH power which heats the minority ( 1 to $3 \%)^{3} \mathrm{He}$ specie. Simulations of the plasma/IC wave interaction and of the collisional slowing down of the created minority ion tail have been performed in [2] considering 
$30 \mathrm{MW}$ of ICRH coupled power and $67 \mathrm{MHz}$ of resonance frequency. Results for the ${ }^{3} \mathrm{He}$ Probability Distribution Functions (PDF) indicate that in such conditions the resonance layer is centred at $\rho=0.28 \mathrm{~cm}$ ( $\rho$ being the square root of the normalized poloidal flux function) and that a large fraction $(\sim 50 \%)$ of the minority ${ }^{3} \mathrm{He}$ ions can be accelerated up to temperatures above the critical energy, thus making electron heating the main energy transfer mechanism [2]. By using a bi-Maxwellian parameterization of the ${ }^{3} \mathrm{He} \mathrm{PDF}$ in terms of $\mathrm{T}_{\perp}$ and $\mathrm{T}_{/ /}$, it is found that the perpendicular temperature of the heated ${ }^{3} \mathrm{He}$ population reaches about $\mathrm{T}_{\perp}=700 \mathrm{keV}$ after $9 \mathrm{~s}$ of applied ICRH power, with a parallel temperature of about $60 \mathrm{keV}$, as shown in Fig. 1. The ICRH power deposition profile is rather peaked, as indicated by the narrow effective temperature profile of Fig. 1. As often happens in the fast ion population accelerated by ICRH, the PDF is strongly anisotropic. This effect is evident from Fig. 2 where one can see that the high energy tail of the PDF changes strongly with the pitch angle $\theta$, being higher for $\theta$ close to $90^{\circ}$. The simulated spatial and energetic ${ }^{3} \mathrm{He}$ PDF has been used as input to predict the spectra observed by the set of selected fast ion diagnostics.

\begin{tabular}{ccc}
\hline & $\begin{array}{c}\text { H mode } \\
\text { reference }\end{array}$ & $\begin{array}{c}\text { H mode } \\
\text { extreme }\end{array}$ \\
\hline $\mathrm{I}_{\mathrm{p}}(\mathrm{MA})$ & 6.5 & 8.0 \\
$\mathrm{q}_{95}$ & 3 & 2.6 \\
$\mathrm{~B}_{\mathrm{T}}(\mathrm{T})$ & 7.5 & 8.5 \\
$\mathrm{H}_{98}$ & 1 & 1 \\
$\mathrm{P}_{\text {th-H }}(\mathrm{MW})$ & $14-18$ & $22-35$ \\
$\tau_{\mathrm{E}}(\mathrm{s})$ & 0.4 & 0.65 \\
$\mathrm{~T}_{0}(\mathrm{keV})$ & 13 & 9.5 \\
$\mathrm{Q}$ & 0.65 & 1.5 \\
$\mathrm{t}_{\text {pulse }}(\mathrm{s})$ & 20 & 13 \\
$\mathrm{t}_{\text {flat }}(\mathrm{s})$ & 13 & 2 \\
$\mathrm{P}_{\mathrm{AUX}}(\mathrm{MW})$ & 30 & 40 \\
\hline
\end{tabular}

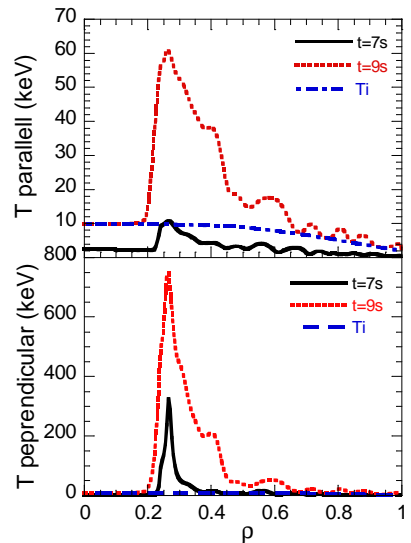

Table 1: FAST H-mode plasma scenarios [1].

Fig. 1: (colour on line) Time evolution of the parallel (top) and perpendicular (bottom) effective ${ }^{3} \mathrm{He}$ temperature versus the normalized radial position $\rho$ for the $S H$ reference plasma. Also shown is the bulk ion temperature (dotted dashed line).

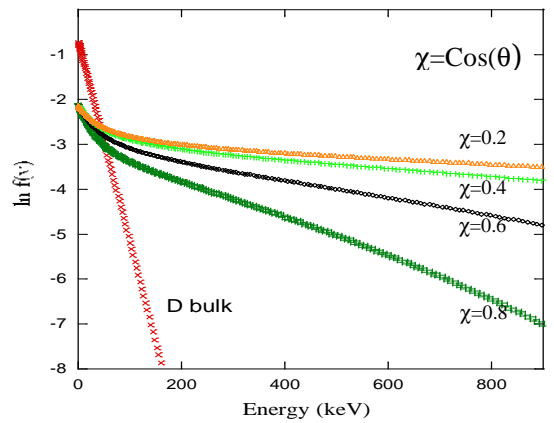

Fig. 2: (colour on line) Simulated steady state ${ }^{3} \mathrm{He}$ PDF $\rho=0.28$ for FAST H-mode reference plasma scenario. The distribution function is shown at different pitch angles $(\theta)$ together with a Maxwellian distribution of bulk D ions.

\section{Diagnosis of fast ${ }^{3} \mathrm{He}$ population}

For the diagnosis of the fast ${ }^{3} \mathrm{He}$ population, the role of possible Line Of Sight (LOS) have been investigated. Two combined LOS for GRS and NES diagnostics have been studied: i) a vertical LOS (looking the plasma from an upper vertical port) at a distance of about $3.5 \mathrm{~m}$ from the plasma core, with a $1 \mathrm{~m}$ long/40mm inner diameter collimator; ii) LOS parallel to the magnetic field, which should be regarded as a limit case for a possible LOS to be inserted on an equatorial port. Three LOS on the equatorial plane are instead proposed for the CTS. In the case of the GRS and NES, the ${ }^{3} \mathrm{He}$ population is modelled with a "cut" anisotropic Maxwellian population of temperature $\mathrm{T}_{3 \mathrm{He}}$ and a Gaussian pitch angle distribution centred at $90^{\circ}$ [3]. The observables are thus the effective tail temperature, $T_{3 \mathrm{He}}$, for NES and GRS diagnostics, and $\left(\mathrm{T}_{\perp}, \mathrm{T}_{/ /}\right)$for the CTS.

\subsection{Gamma ray spectroscopy of ${ }^{3} \mathrm{He}$ ions}

In fusion plasmas gamma ray emission comes from reactions between fast ions and impurities of the type $\mathrm{a}(\mathrm{A}, \mathrm{b}) \mathrm{B}^{*}$, where a indicates the fast ion, $\mathrm{A}$ the impurity ion and $\mathrm{B} *$ the excited nucleus 


\begin{tabular}{cc}
\hline Level & Energy $(\mathrm{keV})$ \\
\hline L1 & 2125 \\
L2 & 4445 \\
L3 & 5020 \\
L4 & 6743 \\
L5 & 6792 \\
L6 & 7286 \\
L7 & 7978 \\
L8 & 8560 \\
L9 & 8920 \\
L10 & 9185 \\
L11 & 9274
\end{tabular}

Table 2: List of the first eleven

${ }^{11} B$ energetic levels.

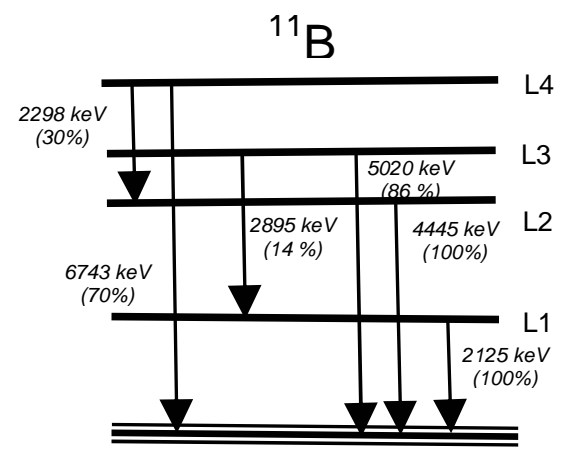

Fig.3 Level scheme for the first four excited levels of ${ }^{11} B$ with branching ratios and yenergies.

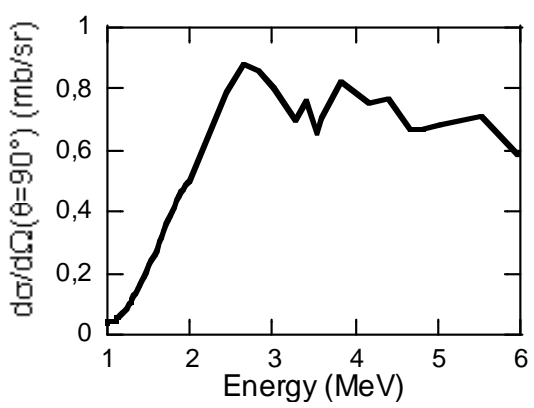

Fig.4 Differential cross section $d \sigma / d \Omega\left(\theta=90^{\circ}\right)$ for populating the third excited state of ${ }^{11} B$.

produced by the reaction [4]. Different kinds of information can be extracted from the measured spectrum. For instance, the identification of characteristic gamma ray peaks is a footprint of fast ions exceeding the threshold energies of the reactions, while peak intensities contain a combined information on both the fast ion density and temperature, beside the impurity concentration. The most detailed level of information is obtained by the Doppler broadened shape of the observed emission lines, since it can provide an independent measurement of the fast ion temperature. Doppler Broadening measurement of fast ions has been proposed about 20 years ago [4], but the observation and interpretation on a fusion plasma is recent [5]. The analysis carried out in [5] has shown that changes in the peak shape strongly depend on the detailed differential cross section of the underlying nuclear reaction.

A study of the cross sections involving energetic ${ }^{3} \mathrm{He}$ ions and ${ }^{9} \mathrm{Be}$ has indicated that reactions of the kind ${ }^{3} \mathrm{He}\left({ }^{9} \mathrm{Be}, \mathrm{x} \gamma\right) \mathrm{Y}$ can be used for diagnosing the ${ }^{3} \mathrm{He}$ population of FAST. The reactions among ${ }^{3} \mathrm{He}$ and ${ }^{12} \mathrm{C}$ impurities, which have been measured at JET in Ref [5] and can provide a measurement of $\mathrm{T}_{3 \mathrm{He}}$, have been discarded since FAST has a Tungsten wall and thus ${ }^{12} \mathrm{C}$ impurities are not present. Also beryllium is not present as impurity in FAST, but it can be evaporated from the walls as done for instance at JET [4]. In particular, the ${ }^{3} \mathrm{He}\left({ }^{9} \mathrm{Be}, \mathrm{p} \gamma\right){ }^{11} \mathrm{~B}$ reaction, has been selected for the present study for the reasons described below. The ${ }^{3} \mathrm{He}\left({ }^{9} \mathrm{Be}, \mathrm{p} \gamma\right){ }^{11} \mathrm{~B}$ reaction is exothermic and its $Q$ value $(10.32 \mathrm{MeV})$ exceeds the energy of the first 14 excited states of ${ }^{11} \mathrm{~B}$ nucleus. Due to the high $\mathrm{Q}$ value several ${ }^{11} \mathrm{~B}$ excited states can be populated by the reaction and yield gamma rays through cascade transitions towards the ground state (See Table 2 and Fig. 3). Despite the high $\mathrm{Q}$ value, the Coulombian repulsion between ${ }^{3} \mathrm{He}$ and ${ }^{9} \mathrm{Be}$ ions makes the cross section for this reaction negligible for $\mathrm{E}_{3 \mathrm{He}}>1 \mathrm{MeV}$. This means that ${ }^{3} \mathrm{He}$ ions in the tail of the energy distribution are main responsible for the gamma ray emission.

Cross section data for this reaction based on accelerator measurements are available in the literature [6] for several ${ }^{3} \mathrm{He}$ laboratory energies. Measurements include both the excitation function $\mathrm{d} \sigma / \mathrm{d} \Omega\left(\theta_{\text {c.m. }}=90^{\circ}\right)$ over the laboratory range $\mathrm{E}_{3 \mathrm{He}}=1-6 \mathrm{MeV}$ and the differential cross section $\mathrm{d} \sigma / \mathrm{d} \Omega\left(\mathrm{E}_{3 \mathrm{He}}, \theta\right)$. Cross section data are available for ${ }^{11} \mathrm{~B}^{*}$ levels up to the $8^{\text {th }}$ excited state, except for gaps in the data for the first two states in the range $\mathrm{E}_{3 \mathrm{He}}<3 \mathrm{MeV}$. Such lack of data for the first two excited states, which are important due to the characteristics cascade $\gamma$ emission, preclude the study of the diagnostic information provided by the relative intensities of the different $\gamma$ peaks. Therefore, in the present study, the $\gamma$-line Doppler broadening has been chosen as the main diagnostic observable to evaluate $\mathrm{T}_{3 \mathrm{He}}$. The $\gamma$ ray line at $5.020 \mathrm{MeV}$ (L3 to ground state transition) has been chosen as the main diagnostic observable to evaluate the effective tail temperature of ${ }^{3} \mathrm{He}$ 


\section{Thermal + HKN}

i) $\mathrm{d}+\mathrm{d} \rightarrow \mathrm{n}+{ }^{3} \mathrm{He}$

$\mathrm{d}+{ }^{3} \mathrm{He} \rightarrow \mathrm{d}^{\prime}+{ }^{3} \mathrm{He}$

ii) d' $+d \rightarrow$ n' $+{ }^{3} \mathrm{He}$

Fig.5 Schematics of the thermal (i) and HKN (ii) components in the neutron spectrum.

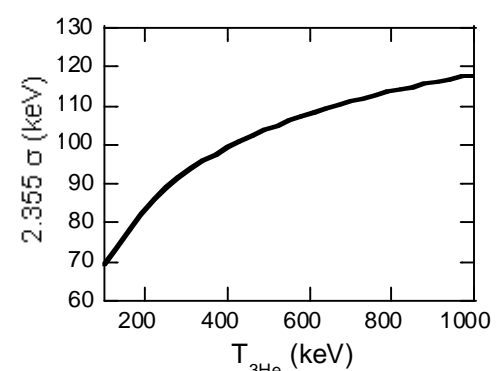

Fig.6 Broadening of the 5.02 $\mathrm{MeV} \gamma$-ray emission peaks from the ${ }^{9} \mathrm{Be}\left({ }^{3} \mathrm{He}, \mathrm{p} \gamma\right)^{11} \mathrm{~B}$ reaction. See text for details.

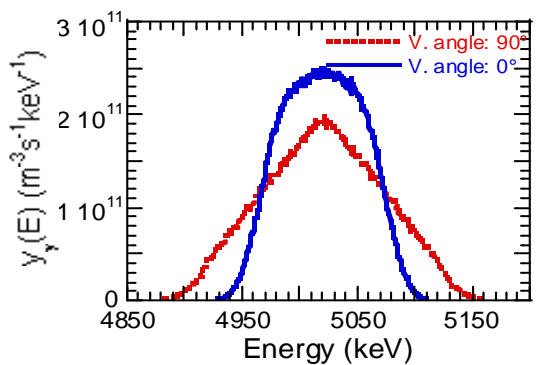

Fig.7 Simulated $5.02 \mathrm{MeV}$-ray emission peak from the ${ }^{9} \mathrm{Be}\left({ }^{3} \mathrm{He}, \mathrm{p} \gamma\right)^{11} \mathrm{~B}$ reaction for the FAST H-mode reference scenario at $\rho=0.28$, for two viewing angles relative to the magnetic field.

ions $\left(\mathrm{T}_{3 \mathrm{He}}\right)$. This transition offers the advantage of a negligible contribution from higher excited states (less than 1\%), good availability of differential cross section and a higher absolute spectral broadening compared to lower $\gamma$ ray energies. A feature of the ${ }^{3} \mathrm{He}\left({ }^{9} \mathrm{Be}, \mathrm{p} \gamma\right){ }^{11} \mathrm{~B}$ excitation function for the L3 state (see Fig. 4) is its mild variation for $\mathrm{E}_{3 \mathrm{He}}>2.5 \mathrm{MeV}$, after a sharp rise between 1 and $2 \mathrm{MeV}$. This is in contrast with reactions which are instead dominated by strong resonances in the cross section, which is a feature identified as a limitation to the possibility of measuring temperature in wide ranges from the Doppler broadened peak shape [5].

A set of simulations have been performed to calculate the $\gamma$ emission spectrum produced by anisotropic Maxwellian populations of ${ }^{3} \mathrm{He}$ ions with different $\mathrm{T}_{3 \mathrm{He}}$ values and a Gaussian pitch angle distribution centred at $90^{\circ}$ with $\mathrm{FWHM}=10^{\circ}$, for a $1 \%{ }^{9} \mathrm{Be}$ concentration. Results shown in Fig. 6 indicate that the $\mathrm{T}_{3 \mathrm{He}}$ dependence of the spectrum broadening $\left(\mathrm{W}_{\gamma}=2.355 \cdot \sigma\right.$ with $\sigma$ standard deviation of the $\gamma$ peak) can be heuristically described by the curve $\mathrm{W}_{\gamma}=25 \cdot\left(\mathrm{T}_{3 \mathrm{He}}\right)^{1 / 4.4}$ (here both $\mathrm{W}_{\gamma}$ and $\mathrm{T}_{3 \mathrm{He}}$ expressed in $\mathrm{keV}$ ). $\mathrm{A} \mathrm{T}_{3 \mathrm{He}}$ increase from 100 to $600 \mathrm{keV}$ corresponds to a change in $\mathrm{W}_{\gamma}$ from 70 to $105 \mathrm{keV}$. Note that the weak variation of $\mathrm{W}_{\gamma}$ with $\mathrm{T}_{3 \mathrm{He}}$ at higher temperature requires that the GRS measurement should be complemented by other type of tail temperature measurements (e.g. NES, see Section 3.2). Simulations have been performed using the ${ }^{3} \mathrm{He}$ PDF for the FAST H-mode reference scenario, at $\rho=0.28$. The $5.02 \mathrm{MeV} \gamma$ spectral shape depends on the relative angle between the LOS and the magnetic field, as is shown in Fig. 7. The observed difference in the $\gamma$ peak shape reflects the anisotropy of the ${ }^{3} \mathrm{He}$ distribution created by the ICRH acceleration mechanism, which provides a preferential energy transfer to the particle perpendicular motion. An additional LOS looking at the plasma with some inclination with respect to B field would be useful to study of the anisotropy of the ${ }^{3} \mathrm{He}$ population.

The measurement of $\mathrm{W}_{\gamma}$ with the needed accuracy $(<10 \%)$ is challenging and requires a high energy resolution gamma ray spectrometer together with a good stability of the spectrometer response function. It is proposed to use $\mathrm{LaBr}_{3}$ scintillation detectors equipped with a digital acquisition system. Such choice combines a good energy resolution ( $\mathrm{R}=50-75 \mathrm{keV}$ at $5 \mathrm{MeV})$ [7] with low sensitivity to neutron background, good detection efficiency and high count rate capability $(>\mathrm{MHz})$ [8]. The $5.02 \mathrm{MeV}$ flux at the detector position along the vertical LOS has been estimated to be about $4 \cdot 10^{5} \gamma \cdot \mathrm{s} \cdot \mathrm{cm}^{-2}$, which provides a time resolution for the measurement of $\mathrm{T}_{3 \mathrm{He}}$ in the range $25-100 \mathrm{~ms}$ with statistical uncertainties in the range 5-10\%. The above estimate is based on an upper limit of the detector count rate capability of $2 \mathrm{MHz}$ [8], a detection efficiency of $30 \%$ at $5 \mathrm{MeV}$ and assuming the ratio $\mathrm{C}_{\gamma 5.02} / \mathrm{C}_{\gamma \text { tot }} \sim 0.1$, where $\mathrm{C}_{\gamma 5.02}$ and $\mathrm{C}_{\gamma \text { tot }}$ are the recorded 


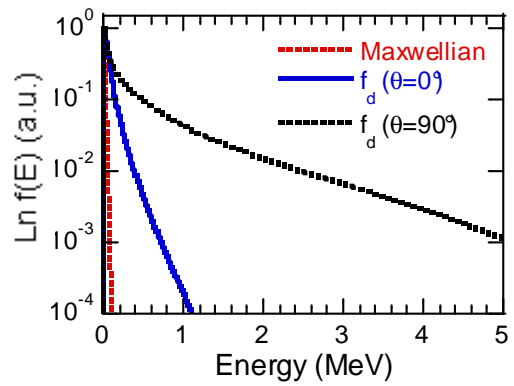

Fig.8 PDF of suprathermal D ions Fig. 9a Simulated neutron emisdue to HKN reactions together sion spectrum for FAST H-mode with a $10 \mathrm{keV}$ Maxwellian, for the reference scenario for two viewFAST H-mode reference scenario. ing angles relative to the magnetic field. Together with the neutron spectrum is shown the thermal component alone.

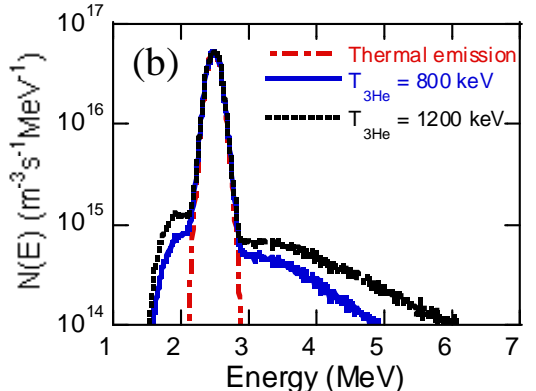

Fig. $9 b$ Simulated thermal emission and HKN neutron spectrum for Maxwellian $1 \%{ }^{3} \mathrm{He}$ ions of 800 and $1200 \mathrm{keV}$ with $\theta=90^{\circ} \pm 10^{\circ}$.

$5.02 \mathrm{MeV}$ and total count rate in the energy range $0.1-10 \mathrm{MeV}$, respectively. The statistical uncertainty on the determination of $\mathrm{T}_{3 \mathrm{He}}$ is due to the uncertainty in the determination of the spectrum broadening, i.e. $\Delta T_{3 H e} / T_{3 H e}=4.4 \cdot \Delta W_{\gamma} / W_{\gamma}$; for a Gaussian spectrum the relation $\Delta W_{\gamma} / W_{\gamma}=(2 N)^{-1 / 2} \cdot\left(1+R^{2} / W^{2}\right)$ holds [9], where $N$ is integral number of counts in the $5.02 \mathrm{MeV}$ peak and $R$ is the spectrometer energy resolution.

\subsection{Neutron emission from ${ }^{3} \mathrm{He}$ knock-on reactions}

In D plasmas neutron emission comes from the fusion reaction $\mathrm{d}+\mathrm{d} \rightarrow \mathrm{n}(2.45 \mathrm{MeV})+{ }^{3} \mathrm{He}(0.82$ $\mathrm{MeV}$ ) and the neutron energy spectrum reflects the energy distribution of the deuteron population. Besides the thermal Maxwellian distribution, the deuteron PDF can present suprathermal populations (d'), caused for instance by external heating. As a result, the neutron spectrum is made of a Gaussian shaped thermal region centred at $2.45 \mathrm{MeV}$ and a suprathermal component, extending to higher neutron energies and resulting from the reactions $d^{\prime}+d$ and $d^{\prime}+d^{\prime}[10]$. In $D$ plasmas with ${ }^{3} \mathrm{He}$ ions present as minority species and ICRH tuned at $\omega_{3 \mathrm{He}}$, fast ${ }^{3} \mathrm{He}$ ions can transfer energy to d ions through knock-on collisions (HKN, see Fig. 5) [11]. These events, though rare, are effective in transferring energy to the deuterons and generate an anisotropic d' population that resembles the anisotropy of the ${ }^{3} \mathrm{He}$ source (see Fig. 8). Simulations of the neutron emission at the centre of the ${ }^{3} \mathrm{He}$ resonance ([11]) have been performed for the FAST Hmode reference scenario for angles of $0^{\circ}$ and $90^{\circ}$ relative to the magnetic field (Fig.9a). The results indicate the presence of a suprathermal HKN component with intensity (above $3 \mathrm{MeV}$ ) $~ 8 \%$ of the thermal emission and an energy tail extending up to 6-8 $\mathrm{MeV}$. As expected the high energy neutron tail becomes wider when passing from $0^{\circ}$ to $90^{\circ}$ due to the anisotropy of the d' population that has been preferentially accelerated perpendicularly to the magnetic field.

From the broadening of the thermal component the bulk $\mathrm{D}$ ion temperature $\mathrm{T}_{\mathrm{i}}$ can be inferred via the well known relation: $\mathrm{W}_{\mathrm{n}}=82.5 \cdot\left(\mathrm{T}_{\mathrm{i}}\right)^{1 / 2}$. The slope and intensity of the HKN part of the neutron spectrum is instead related to $\mathrm{T}_{3 \mathrm{He}}$. It is interesting to note that the HKN measurements can well complement GRS measurements for $\mathrm{T}_{3 \mathrm{He}}>600 \mathrm{keV}$, since the HKN signal increases (higher intensity and wider tails) with increasing $\mathrm{T}_{3 \mathrm{He}}$ as shown in Fig.9b for the case of single Maxwellian ${ }^{3} \mathrm{He}$ PDF. A more detailed study of the dependence of the HKN component with $\mathrm{T}_{3 \mathrm{He}}$ is still in progress. 


\section{EXW/P7-26}

Several neutron spectrometers have been developed for NES measurements on D fusion plasmas. For $\mathrm{T}_{\mathrm{i}}$ measurements time-of-flight (TOF) neutron spectrometer and compact spectrometers can be used. An estimate of the $2.5 \mathrm{MeV}$ neutron flux at the proposed NES detector position in the vertical port yields a value of $\sim 1.8 \cdot 10^{8} \mathrm{n} \cdot \mathrm{s} \cdot \mathrm{cm}^{-2}$; with such flux, a time resolution on $\mathrm{T}_{\mathrm{i}}$ in the range 20-40 ms with statistical uncertainty $\leq 5 \%$ is expected (TOF case [12]). For the detection of the HKN component, neutron spectrometers featuring lower detection efficiency but an higher sensitivity to weak components are needed, such as for instance those based on thin foil neutron to proton converter [13]. From the predicted HKN flux and given the spectrometer efficiencies $\left(\sim 10^{-4}\right)$ a preliminary estimate of time resolution on $\mathrm{T}_{3 \mathrm{He}}$ of $25-100 \mathrm{~ms}$ with $5-10 \%$ statistical accuracy is expected.

\subsection{Collective Thomson scattering}

The Collective Thomson Scattering (CTS) is an active diagnostic in which the high-power beam generated by a mm-wave gyrotron is injected into the plasma and the scattered 'beam' propagating along one or more LOSs is collected. The information on the ion velocity distribution comes from the Doppler broadening of the scattered 'beam'. CTS diagnostic will provide space- and time- resolved information on the dynamics of the fast ion population generated by ICRH. The configuration proposed for FAST is similar to the one foreseen for ITER [14] and basically consists in a backscattering geometry (transmitting and receiving antennas accommodated in a single port) with propagation below the fundamental Electron Cyclotron (EC) resonance. The CTS spectra broadening depends on both the scattering angle and the unidimensional ion velocity distribution along the scattering vector direction, defined by the difference of wave-vectors of the scattered and incident beams. Two different receiving LOS will be used to characterize the parallel/perpendicular components of the anisotropic PDF of the fast ion tails. The spatial profile of the ion tails will be also obtainable thanks to a wide scanning capability of the receiving antennas. The proposed geometry has been studied in view of the plasma accessibility, the sensitivity to refraction and low level of background EC emission. It features three LOS from an equatorial port for the millimetric-beam antennas. The one for the probe is directed from the port extreme (right) edge near the equatorial plane aiming at the plasma centre with a toroidal angle around $20^{\circ}$ (to the left). In Fig.10 the side and top views of the FAST vacuum vessel shows the projection of the probe beam and the lines for receiving the scattered radiation from the centre and from the plasma periphery (the projections, in two shades of green, results superimposed in side view). The beam trajectories have been evaluated with ray tracing using the code SPECE [15] for different frequencies (see Figs.11 and 12) and are not very sensitive to the frequency, as required for collecting a scattered radiation over a spectrum a few $\mathrm{GHz}$ wide, avoiding spectral distortions. The toroidal angle of $-20^{\circ}$ is aimed at avoiding the cut-off found already for an angle of $30^{\circ}$ (see Fig.13). The first receiving antenna is vertically displaced, with a scanning capability in the toroidal and poloidal directions in order to keep the alignment when moved along the probe beam. In such a way the spatial distribution of scattered radiation can be analyzed with a scattering vector forming angles with respect to the magnetic field direction of around $125^{\circ}$. For a given magnetic field direction, the sign of the toroidal angle of the received beam influences the sensitivity of refraction to frequency and ECE background level. As seen in Fig.11, while oblique rays at different frequencies, chosen between 100 and $120 \mathrm{GHz}$ in steps of two, exhibit almost the same trajectory for $-20^{\circ}$ (red lines in Fig.10) they show a wider spread e for the $+20^{\circ}$ (blue lines). Moreover, as will be shown below, the level of scattered radiation that is much lower for $-20^{\circ}$. The second of the two receiving antennas will be placed at the edge of the port that is toroidally opposite to the first one. When scanning spatially the probe beam, the angles described by the scattering vector 
EXW/P7-26

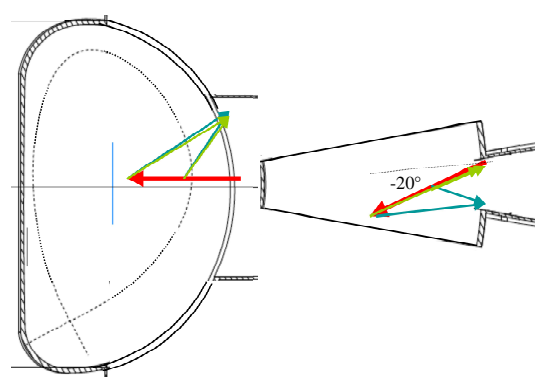

Fig.10: Side (left) and top (right) projections of the ray trajectories for the probing (red) and scattered (green) 'beams'. Some overlap is present in the side view.

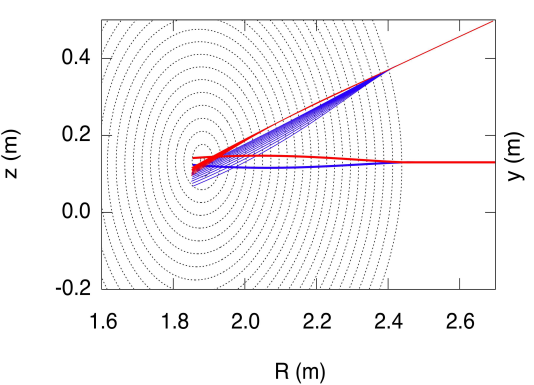

Fig. 11: Ray trajectories in the $100-120 \mathrm{GHz}$ range. Rays at $-20^{\circ}$ toroidal angle (red) exhibit a smaller spread compared with the ones at $+20^{\circ}$ (blue lines).

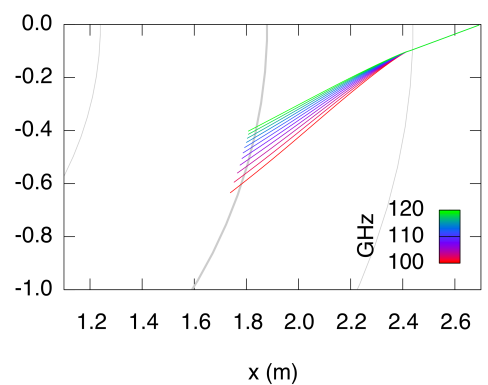

Fig. 12: Projection on the equatorial plane of the ray trajectories in the $100-120 \mathrm{GHz}$ range assumed launched from one of the receiving antennas.

with respect to the magnetic field span from $110^{\circ}$ to about $95^{\circ}$ (from the center to the plasma edge). Propagation in the direction perpendicular to the magnetic field, where the spectra might be affected by modulations, is in this way avoided. Furthermore, sufficient margins are available to recognise anisotropies of the velocity distribution of the fast ions by comparison with results provided by the first receiving antenna (see Fig.10). The frequency of the source and the power level required to optimize the Signal to Noise $(\mathrm{S} / \mathrm{N})$ ratio have been investigated and compared to the background EC emission, the main source of noise in CTS. Estimates performed with the same SPECE code [15] for the X mode required to operate CTS at the very high densities of FAST plasmas, indicate that a source frequency above $100 \mathrm{GHz}$ will be needed to avoid strong refraction. In Fig.14 the ECE background level for the plasma parameters of interest to the FAST $\mathrm{H}$-mode reference scenario [2] is shown as a function of frequency, for LOSs respectively perpendicular, oriented at $\pm 20^{\circ}$ in the equatorial plane and oblique (up-directed). As already anticipated, a significant difference is seen in terms of ECE background between the propagation at $+20^{\circ}$ and that at $-20^{\circ}$. This lower background level was the main motivation for the choice of the minus direction for the toroidal receiving line.

The ECE background level has to be compared with the two components of the scattered signal associated respectively to the bulk ions and the fast ${ }^{3} \mathrm{He}$ ions. While much stronger, the signal due to the bulk ions is limited to a (double-sided) bandwidth of $\pm 1.5 \mathrm{GHz}$ about the probe frequency. The weaker signal due to the fast ions instead extends to a (single-sided) bandwidth of $8 \mathrm{GHz}$, above which the electron feature becomes dominant in the spectrum. A bandwidth as wide as 6.5 $\mathrm{GHz}$ is therefore available for the CTS measurements. Simulations of the CTS spectra and estimates of the $\mathrm{S} / \mathrm{N}$ have been made accounting for power levels of $1 \mathrm{MW}$, as presently deliverable by mm-wave gyrotrons, and assuming optimized collection of the scattered radiation. As shown in Fig. 15, while the bulk ion spectrum is expected to overcome the ECE background for probe frequencies up to $115 \mathrm{GHz}$, above $100 \mathrm{GHz}$, the minimum frequency for avoiding cut-offs, the fast-ion feature is always below the ECE background. Given that the ECE emission cannot be regarded as constant over the required spectral range and thus cannot be easily subtracted, modulation of the probe power in connection with synchronous detection has to be used to discriminate the scattering signal from the background. The $S / N$ in a representative channel is readily estimated using the formula $S / N=T_{S} /\left(T S+T n / \mathcal{E}+T_{E C E}\right) \cdot(B \tau)^{1 / 2}[16]$ where $T n$ is the noise equivalent power of the receiver $(\mathrm{Tn} \sim 1 \mathrm{eV}), \varepsilon$ is the heterodyne efficiency $(\sim 0.5), \mathrm{T}_{\mathrm{ECE}}$ is the ECE radiation background temperature, $\mathrm{B}$ is the bandwidth of the channel of the filter bank, $\tau$ is the integration 


\section{EXW/P7-26}

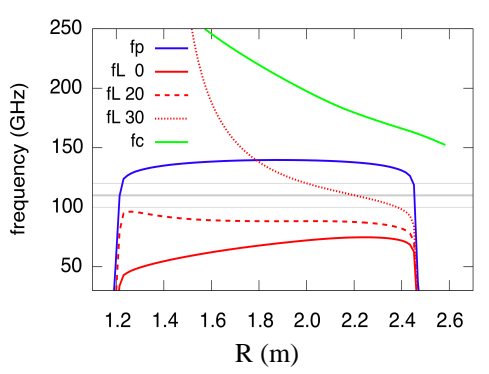

Fig.13: EC resonance frequency $\left(f_{c}\right)$ and cut-offs $\left(f_{p}, f_{L}\right)$ evaluated on the plasma equatorial plane as a function of major radius $R$ for different toroidal angles in FAST conditions.

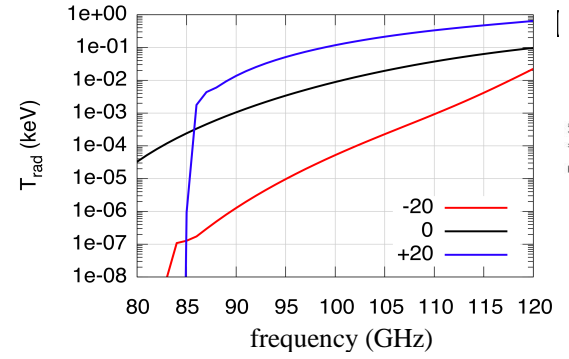

Fig. 14: Expected ECE radiation temperature seen by the receiving antenna for different viewing angles (perpendicular, $\pm 20^{\circ}$ with respect to the radial direction and oblique, up-directed).

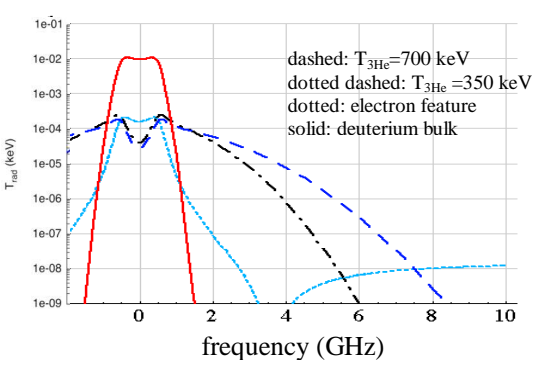

Fig. 15: Expected CTS spectrum showing bulk, electron and fast ion spectra for a $0.5 \%{ }^{3} \mathrm{He}$ minority tail, modeled with maxwellian $P D F$ with $T=700 \mathrm{keV}$ and $T=350$ $\mathrm{keV}$, respectively.

time of about $10 \mathrm{~ms}$, a S/N above unity is found already for frequencies between $100 \mathrm{GHz}$ and $120 \mathrm{GHz}$. A standard mm-wave gyrotron with $1 \mathrm{MW}$ nominal power and a frequency of 110 $\mathrm{GHz}$ should therefore be suited to the requirements of FAST, offering the possibility to carry out detailed studies of the fast ion dynamics with a spatial resolution in the range $5-10 \mathrm{~cm}$.

\section{Conclusions}

A first assessment of the extent to which fast ion populations can be diagnosed in FAST with Neutron Emission Spectroscopy, Gamma-Ray Spectroscopy and Collective Thomson Scattering has been presented in the present paper. Starting from numerical simulations of the spatial and energetic distribution function of the ICRH-accelerated minority ${ }^{3} \mathrm{He}$ ions, the corresponding spectra observed by the set of diagnostics have been simulated and their sensitivity to the fast ion tail discussed. Results suggest that with suitable line of sights and combining the information provided by the three diagnostics, the temperature of the ${ }^{3} \mathrm{He}$ population can be diagnosed with time resolution in the range $10-100 \mathrm{~ms}$ and $5-10 \mathrm{~cm}$ spatial resolution.

\section{References}

[1] PIZZUTO, A., et al., "The Fusion Advanced Studies Torus (FAST): a proposal for an ITER satellite facility in support of the development of fusion energy", submitted to Nucl Fusion (2009).

[2] CARDINALI, A., et al., Nucl Fusion 49 (2009) 095020.

[3] TARDOCCHI, M., et al.,, Rev. Sci. Instrum. 7977 (2006) 126107

[4] KIPTILY, V. G., et al., Plasma Phys. and Contr. Fusion 48 (2006) R59.

[5] TARDOCCHI, M., et al., "Spectral broadening of characteristic $\gamma$ ray emission peaks from ${ }^{12} \mathrm{C}\left({ }^{3} \mathrm{He}, \mathrm{p} \gamma\right)^{14} \mathrm{~N}$ reactions in fusion plasmas", submittted to Phys. Rev. Letters (2010).

[6] LIN, C.S. et al., J. Phys. 19 (1981) 99; W.R. Coker et al., Nucl. Phys. A91 (1967) 97.

[7] NOCENTE, M., et al., "Energy resolution of gamma-ray spectroscopy of JET plasmas with a $\mathrm{LaBr}_{3}$ scintillator detector and digital data acquisition", to appear on Rev. Sci. Instr. (2010).

[8] TARDOCCHI, M., et al.,, Rev. Sci. Instrum. 79 (2008) 10E524.
[9] TARDOCCHI, M., et al.,., Rev. Sci. Instrum. 75 (2004) 661.

[10] TARDOCCHI, M., et al., Nucl. Fusion 42, 1273 (2002).

[11] NOCENTE, M., et al., Nuclear Fusion 50 (2010) 022001; "Effetti cinetici nello spettro dei neutroni in plasmi $\left({ }^{3} \mathrm{He}\right) \mathrm{D}$ riscaldati a radiofrequenza", M. Nocente, Thesis for the Degree of Master of Science MilanoBicocca University, Italy

[12] GATU JHONSON, M., et al., Nucl. Instr. and Meth. A. 591 (2008) 417.

[13] KAELlNE, J., et al., Phys. Rev. Lett. 85, 1246 (2000).

[14]BINDSLEV H., et al. "ITER Fast Ion Collective Thomson Scattering" Feasibility study, Report on EFDA contract 01.654; ORSITTO, F., GIRUZZI, G., Nucl Fusion 37 (1997) 915.

[15] FARINA, D., et al., AIP Conf. Proc. 988 (2008) 128.

[16] WILSON, T. L. al. "Tools of Radio Astronomy", $5^{\text {th }}$ ed. Springer Verlag (2009). 